\title{
Jeunes filles scoliotiques et orthopédie à la Belle Epoque: l'essor urbain de la mécanothérapie à Lausanne et Genève
}

Mariama Kaba

\section{Summary}

In Belle Epoque towns marked by the industrial and medical surge, a new technical therapy, called mechanotherapy, emerged, stemming from Swedish medical gymnastics and auxiliary to orthopaedics. Aiming mostly at treating scoliosis, this therapy by movement attracted a sizeable female clientele to these towns, because of the hygienic and social conceptions feeding collective imagination linked to the bodies of scoliotic young girls. Taking the Frenchspeaking Swiss towns of Lausanne and Geneva as examples, the article first seeks to describe the emergence of mechanotherapy as a medical and urban phenomenon. It then addresses the role played by scoliosis in this orthopaedic practice, and examines the clientele attracted to the towns, among which well-born young girls seem to be predominant.

Keywords: mechanotherapy, orthopaedics, gymnastics, scoliosis, gender, $19^{\text {th }}$ century medicine

\section{Résumé}

Dans les villes de la Belle Epoque marquées par l'essor industriel et médical, émerge une nouvelle thérapie technique issue de la gymnastique médicale suédoise et subsidiaire à l'orthopédie, appelée mécanothérapie. Destinée en grande partie au traitement de la scoliose, cette thérapie par le mouvement attire en ville une importante clientèle féminine, en vertu des conceptions hygiénistes et sociales qui alimentent l'imaginaire collectif relatif au corps

Dr Mariama Kaba, Institut universitaire d'histoire de la médecine et de la santé publique, Lausanne (mariama_kaba@hotmail.com). 
des jeunes filles scoliotiques. En prenant les exemples suisses romands de Lausanne et Genève, l'article décrit d'abord l'émergence du phénomène médical et urbain qu'est la mécanothérapie. Il s'intéresse ensuite au rôle que joue la scoliose dans cette pratique orthopédique, puis s'interroge sur la clientèle qu'elle attire dans les villes, les jeunes filles de bonne famille semblant occuper une place de choix.

L'histoire de l'orthopédie est traversée par des discours et des pratiques médicales autour d'une affection particulièrement centrée sur les filles, la scoliose. Rappelons qu'à l'origine du terme orthopédie (du grec orthos «droit», et pais, paidos «enfant») se trouve une volonté de vulgarisation pédagogique avant la lettre. Le chirurgien français Nicolas Andry (16581742) emploie ce terme pour la première fois en 1741 dans un ouvrage au titre explicite: L'Orthopédie ou l'art de prévenir et de corriger dans les enfans [sic], les difformités du corps. Le tout par des moyens à la portée des pères et des mères, et de toutes les personnes qui ont des enfans à élever. ${ }^{1}$ Quarante ans plus tard, l'orthopédie clinique est instituée en Suisse par le médecin vaudois Jean-André Venel (1740-1791), qui fonde à Orbe le premier établissement pour le traitement des cas orthopédiques, sans interventions chirurgicales. Quant à la scoliose (du grec skolios «oblique, sinueux»), elle est, comme la plupart des affections orthopédiques, connue depuis l'Antiquité au moins: le vocable est employé par les médecins hippocratiques puis Galien pour désigner les diverses courbures ou inflexions de la colonne vertébrale, et particulièrement sa déviation latérale. Les causes et les traitements en sont multiples, et font l'objet de nombreux débats à travers les siècles. ${ }^{2}$ La corrélation entre scoliose et genre féminin est notable dès les pionniers de l'orthopédie de la seconde moitié du siècle des Lumières:l'affection est souvent citée pour vanter les mérites de telle thérapie orthopédique, ayant pour corollaire une multiplication des discours et des pratiques orthopédiques autour du corps des jeunes filles scoliotiques. ${ }^{3}$ Notons qu'à l'heure actuelle, on reconnaît que la proportion des scolioses selon les sexes est de 3,5 filles pour 1 garçon. On s'accorde également à qualifier la scoliose $\mathrm{d}^{\prime}$ «idiopathique» (dont la cause n'est pas connue) pour environ $75 \%$ des cas. Son origine serait essentiellement multifactorielle (facteurs génétiques, hormonaux, neurologiques, biomécaniques, de croissance, troubles du métabolisme).$^{4}$

1 Sur l'orthopédie d'Andry, voir Vigarello 2001,17sq.

2 Voir par exemple Rang 2000, 143-170; Valentin 1961, 9-36 et 61-63.

3 Sur ce sujet particulier et pour un développement substantiel, voir ma thèse de doctorat:Kaba 2011. Sur le sujet du corps sexué et de ses déficiences, voir aussi Kaba 2006a; Kaba 2006b.

4 Campagne 2000; Hefti 1998, 73. 
Durant la Belle Epoque, sous les effets de l'industrialisation et de l'essor médical, l'orthopédie se dote d'une nouvelle thérapie mécanisée qui se destine notamment au traitement des scolioses par le mouvement, appelée mécanothérapie. En prenant comme exemples les villes de Lausanne et de Genève, respectivement chefs-lieux des cantons suisses romands de Vaud et de Genève et pionnières en matière de mécanothérapie dans l'espace francophone, cet article décrit l'émergence de ce phénomène médical particulier aux centres urbains. Il s'intéresse ensuite au rôle que joue la scoliose dans ce nouveau secteur orthopédique, puis s'interroge sur la clientèle auquel il se destine, les pratiques et discours médicaux semblant surtout cibler les jeunes filles scoliotiques de bonne famille.

\section{L'essor de la mécanothérapie à la Belle Epoque, reflet de l'impact de l'industrialisation urbaine sur l'orthopédie}

Dans le contexte de renouveau hygiéniste propre au XIX ${ }^{\mathrm{e}}$ siècle, qui accorde notamment une plus grande place au mouvement corporel, se développe un «système complet de gymnastique raisonnée» ${ }^{5}$ appelé gymnastique suédoise, comportant un versant militaire, pédagogique et médical. Ce système est l'œuvre du Suédois Pehr Henrik Ling (1776-1839), maître d'escrime et pédagogue de la danse, nommé en 1813 directeur du premier Institut central - ou royal - de gymnastique de Stockholm, fondé par décret royal. Dans le versant médical de la gymnastique de Ling, certains exercices sont empruntés à la gymnastique pédagogique et intégrés dans un système complexe, combinant 75 positions avec une trentaine de mouvements (flexion, extension, torsion, étirement, roulement, écartement, rapprochement, soulèvement, balancement, pression, tension, etc.). Ils sont dits actifs, passifs ou de résistance, et sont effectués pour certains avec l'aide d'un médecin ou d'une personne formée en la matière, le gymnaste. ${ }^{6}$

La diffusion de la gymnastique suédoise prend une ampleur internationale et connaît un nouvel essor dans la seconde moitié du XIX ${ }^{\mathrm{e}}$ siècle, par le biais de la mécanothérapie. En 1857, l'orthopédiste suédois Gustav Zander (18351920) a en effet l'idée de concevoir des appareils qui imitent la méthode manuelle de Ling: la main du médecin ou du gymnaste est remplacée par des machines, avec lesquelles peuvent être exécutés aussi bien les mouvements passifs que les mouvements de résistance. En 1865, Zander fonde à Stockholm le premier Institut médico-mécanique et exporte son système, véritable

5 Reymond 1893, 685.

6 Ibid., 685-686; Scholder 1897, 17-18. 
produit de la ville industrieuse favorisé par le transfert technologique de la mécanique et répondant aux critères économiques de standardisation et de rationalisation - un phénomène également observé dans d'autres domaines de la médecine, tels que la chirurgie. ${ }^{7}$ Les contemporains sont conscients des arguments modernistes propres à la Belle Epoque qui suscitent l'engouement pour la nouvelle technique, à l'instar de l'orthopédiste Camille Reymond (1857-1905), initiateur de la méthode Zander à Genève:

\footnotetext{
Frappé de quelques inconvénients de la gymnastique manuelle (nécessité d'un grand nombre de gymnastes bien exercés et, partant, prix élevé du traitement), le Dr Gustave Zander eut l'idée de remplacer le gymnaste par des machines. Il fit construire des chefs-d'œuvre d'ingéniosité mécanique au moyen desquels le patient prend ses mouvements. Trente-six machines dosant la résistance avec l'exactitude la plus absolue servent aux mouvements actifs et dixhuit autres, mues par la vapeur ou par un moteur quelconque, donnent les mouvements passifs et les manipulations du massage...

La gymnastique de Zander ne constitue pas un système à part, c'est simplement un autre mode d'application des mouvements de Ling, mode moins compliqué, moins encombré d'aides souvent difficiles à se procurer, véritable article d'exportation appelé à vulgariser la gymnastique de résistance. Aussi voyons-nous des gymnases médico-mécaniques s'établir dans presque tous les grands centres européens et $\mathrm{y}$ fonctionner avec un succès chaque jour croissant. $^{8}$
}

Par le système mécanisé de Zander, qui précède de peu le management scientifique de Taylor, une formation humaine est ainsi épargnée, remplacée par une technique reproduite et diffusée à travers le monde: le savoir-faire du gymnaste est éliminé au profit de celui de l'ingénieur qui conçoit et fabrique les machines médico-mécaniques, tandis que le médecin peut surveiller, seul, un grand nombre de patient.e.s soumis·e.s simultanément à des exercices différents. L'idée est aussi défendue que la machine doit pouvoir, bien mieux que le gymnaste, répéter à l'infini et à tout moment les gestes appliqués aux patient·e·s, en évitant les erreurs humaines découlant de la fatigabilité et de la perte d'attention; cette assurance, ajoutée au fait que la machine peut être interrompue par son utilisateur/-trice dès qu'il/elle le souhaite, doit au final bénéficier pleinement à celui/celle-ci. Ainsi le conçoit l'orthopédiste lausannois Charles Scholder (1861-1918), autre initiateur romand de la méthode médico-mécanique. ${ }^{9}$

Cette méthode va gagner les principales villes européennes par la diffusion des premiers essais et expériences de Zander parus en 1871 dans un ouvrage suédois, traduit en anglais en 1876, puis en allemand en 1879 sous le

7 Ainsi, P.-Y. Donzé note que «la pratique chirurgicale [...] tend à se mécaniser fortement dans les années 1890-1900 avec l'adoption de nouveaux instruments chirurgicaux, de nouvelles tables d'opérations et de nouveaux types de stérilisateurs. Le recours à divers objets techniques produits en série entraîne une standardisation de la pratique chirurgicale, qui favorise à son tour le passage à une chirurgie de masse»: Donzé 2007, 30.

8 Reymond 1893, 690-691.

9 Scholder 1897, 21-24. 
titre Die Zandersche Gymnastik und das mechanisch heilgymnastische Institut in Stockholm. Dans les deux dernières décennies du siècle, ce sont près d'une centaine d'instituts entièrement ou partiellement dédiés à la mécanothérapie qui voient le jour, surtout dans les villes de Suède et d'Allemagne, mais aussi en Autriche, en Suisse, en France, en Silésie prussienne, en Russie, aux Etats-Unis, dans les Indes néerlandaises ou encore au Chili; dans plusieurs endroits, des appareils sont aussi fournis pour l'usage privé. ${ }^{10}$

Les orthopédistes romands Scholder et Reymond, formés en Suède chez les successeurs de Ling et de Zander, sont alors les pionniers de l'implantation de la mécanothérapie dans l'espace géographique francophone, avec leurs instituts médico-mécaniques et orthopédiques fondés respectivement à Lausanne en 1896 et à Genève en $1898^{11}$ (fig. 1). Ce sont aussi durant la Belle Epoque les seuls instituts médico-mécaniques de Suisse romande, du fait du contexte académique et médical propre à cette région: d'une part, la naissance de l'orthopédie clinique en terre vaudoise maintient l'ascendant du canton sur l'orthopédie tout au long du XIX ${ }^{\mathrm{e}}$ siècle; d'autre part, Genève et Lausanne se distinguent comme seules villes universitaires romandes leurs Facultés de médecine étant respectivement fondées en 1876 et 1890 -, canalisant le savoir académique et scientifique médical de la région. A ce facteur stimulant l'essor institutionnel urbain s'ajoutent ceux de la densité démographique et de l'expansion du tourisme médical, favorisés par le développement des transports (chemin de fer, bateau à vapeur), Lausanne et Genève figurant alors parmi les villes les plus attractives des bords du Lac Léman. Enfin, la mécanothérapie constitue une niche professionnelle innovante sur un marché médical en pleine expansion, la démocratisation des études attirant de nombreux praticiens qui proviennent de couches sociales moins élitistes qu'autrefois. ${ }^{12}$

Thérapie neuve, la mécanothérapie est néanmoins rattachée à d'anciennes techniques telles que massage, gymnastique et manipulations orthopédiques, développées au sein de la gymnastique médicale suédoise. Etant une adaptation de celle-ci, les troubles traités sont sensiblement identiques d'une thérapie à l'autre. Ainsi, les instituts médico-mécaniques et orthopédiques de Scholder et Reymond se destinent autant aux troubles de la locomotion consécutifs à un accident ou à une opération qu'aux troubles de l'assimilation (anémie, chlorose, faiblesse), en passant par les troubles digestifs, les

10 Ibid., 19-20 et 42.

11 A l'exception d'une tentative avortée de l'usage de machines de Zander dans un gymnase parisien en 1879, l'implantation d'instituts médico-mécaniques en France est plus tardive: Monet 2009, 230 sq.

12 Sur l'expansion de la médecine en Suisse romande à la Belle Epoque, voir Donzé 2007, Rieder 2009. 


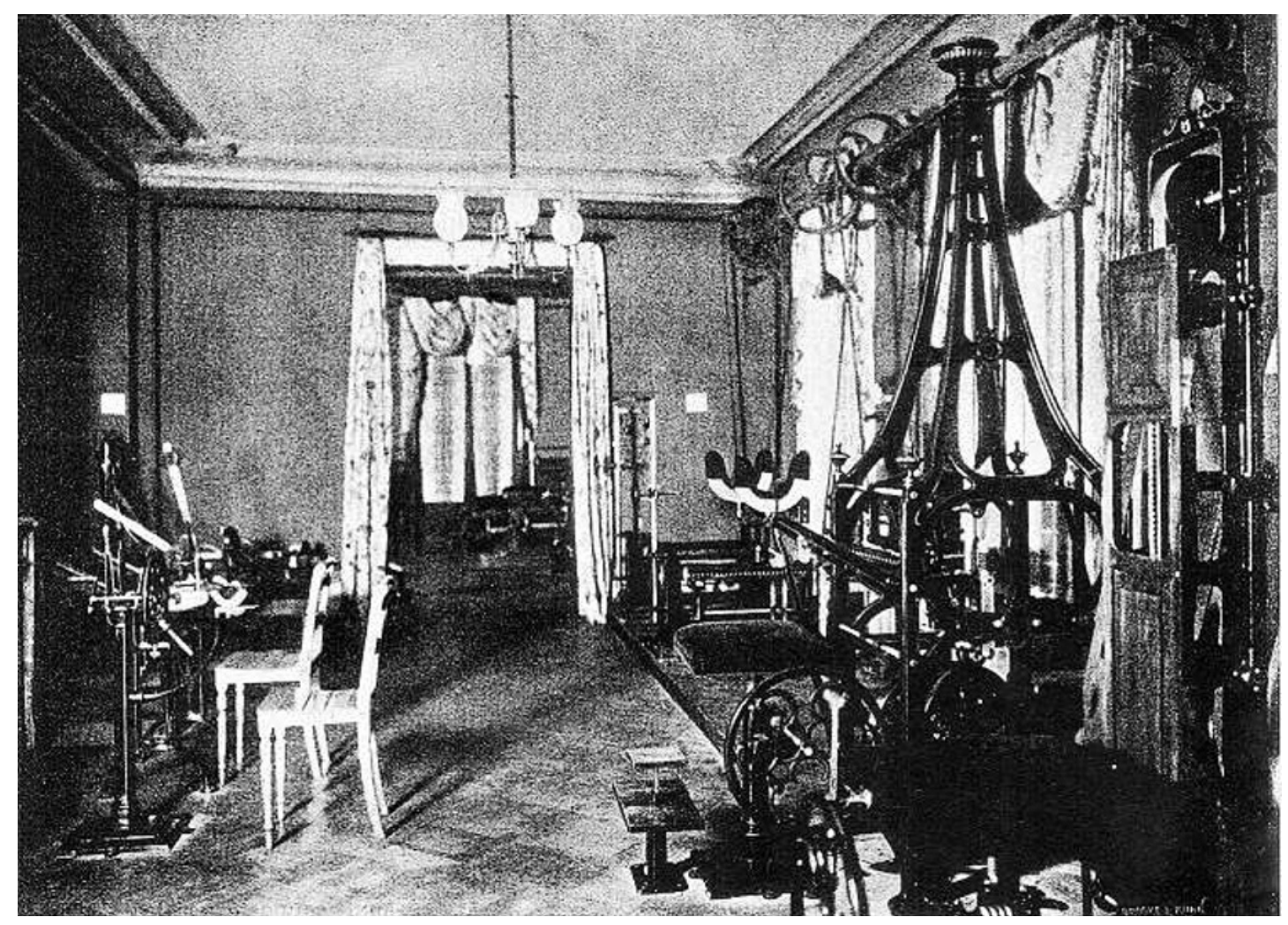

Fig.1: Institut médico-mécanique et orthopédique du Dr C. Reymond, à Genève (Bonna 1899, 45). C'est en 1898 que le Dr Reymond ouvre cet institut à Genève, le second de Suisse romande après celui du Dr Scholder, fondé en 1896 à Lausanne.

maladies nerveuses, celles du cœur, des voies respiratoires, de la nutrition, ou du sexe féminin. ${ }^{13}$ En outre, la mécanothérapie se conçoit aussi comme une «gymnastique hygiénique», qui «convient [...] à tous ceux qui ont besoin de se fortifier et pour lesquels les formes ordinaires de la gymnastique seraient trop fatigantes ou trop difficiles». ${ }^{14}$

A l'instar de la gymnastique suédoise qu'elles sont censées remplacer, les machines de Zander semblent donc avoir la capacité de soigner par le mouvement une large palette de maux, au-delà des affections orthopédiques habituelles. L'orthopédiste de la fin du XIX ${ }^{\mathrm{e}}$ siècle qui, à l'instar de Scholder ou de Reymond, s'empare de la mécanothérapie serait un peu comme le chirurgien de l'époque: une sorte de généraliste capable, par son savoir-faire éclectique inspiré des nouvelles technologies, de soigner tant les affections internes qu'externes. Cependant, parmi l'ensemble des maladies auxquelles se destine la gymnastique médicale suédoise, manuelle et surtout mécanique, c'est la scoliose qui remporte la palme.

13 Scholder 1897, 27-40; Reymond 1901, 197.

14 Reymond $1898,2^{\mathrm{e}}$ page du prospectus inséré (souligné dans le texte). 


\section{L’incidence de la scoliose dans la mécanothérapie: à propos du corps féminin en péril}

Le concepteur de la mécanothérapie est lui-même à l'origine de la destination particulière des appareils médico-mécaniques pour les patient·e·s scoliotiques: à côté de son institut médico-mécanique, Zander a en effet ouvert un institut orthopédique, où il traite exclusivement les déviations de la colonne vertébrale. Il a aussi décrit ses observations sur le traitement de la scoliose idiopathique dans un texte paru en 1894, intitulé Über die habituelle Skoliose. Aussi, la mécanothérapie appliquée à la scoliose est-elle la plus courante, comme le souligne Scholder:

C'est avec une prédilection toute particulière que le Dr Zander s'est occupé des déviations de la colonne vertébrale dans la construction de ses appareils. Si les autres appareils ne sont pas encore connus partout, il n'en est pas de même de ceux qui ont trait à l'orthopédie et on les trouve mentionnés dans la plupart des manuels.

Le traitement des déviations de l'épine dorsale est, en effet, le point lumineux du système Zander. Autrefois, deux méthodes se trouvaient en présence. Les orthopédistes cherchaient à redresser le squelette au moyen d'appareils exerçant sur le malade une pression correctrice, tandis que d'autres, au contraire, se proposaient uniquement de fortifier les muscles du dos. Zander a mis fin à la dispute en combinant les deux systèmes et l'orthopédie moderne marche dans la même voie que lui. ${ }^{15}$

En l'absence de statistiques comparant la proportion entre les scolioses et les autres affections traitées par la mécanothérapie, on doit se fier aux observations médicales des contemporains pour constater l'incidence de la scoliose dans l'essor de la nouvelle thérapie technique. Ainsi, Reymond confirme aussi que la méthode Zander offre une des meilleures «gymnastiques orthopédiques» consacrées à la scoliose, pour laquelle il souligne que, «des maladies des organes locomoteurs, celle que nous avons eu le plus fréquemment à traiter est la scoliose». ${ }^{16}$

Ces commentaires apportent un élément intéressant aux conceptions de l'orthopédie, laquelle, si on suit le raisonnement de Scholder et de Reymond, s'appliquerait pour une grande part à la scoliose. Ainsi, le lien explicite entre scoliose et orthopédie, occasionnellement établi depuis la fin du XVIII ${ }^{\mathrm{e}}$ siècle, s'est maintenu et se renforce à la fin du siècle suivant. Parangon du traitement de la déviation de la colonne vertébrale, la mécanothérapie est en outre présentée comme ayant l'avantage de concilier - réconcilier - deux écoles orthopédiques, celle de l'immobilisation et celle de la fortification: palliant la méthode du corset rigide qui immobilise la taille déviée dans le but de la redresser, elle vise le même objectif redresseur en fortifiant les muscles par le mouvement. Aussi, Reymond, pourtant ancien adepte du corset, peut-il se targuer d'avoir traité 66 cas de scolioses dans son Institut 15 Scholder 1897, 40.

16 Reymond 1901, 201. 
médico-mécanique de Genève, entre novembre 1898 et janvier 1901. Les patient·e-s se répartissent entre 58 filles et 8 garçons, la moyenne d'âge étant de 14,5 ans. ${ }^{17}$ La prépondérance des jeunes filles scoliotiques traitées dans les instituts médico-mécaniques est-elle alors la norme?

Il faut resituer cette question dans le contexte plus général de la destination de la gymnastique suédoise, d'une part, et des débats sur l'orthopédie et la scoliose des jeunes filles, d'autre part. Concernant le premier point, on précisera que Reymond et Scholder font partie des premiers médecins romands qui partent étudier en Suède la gymnastique suédoise. En 1893, Reymond relève avoir traité le nombre élevé de 117 scolioses par cette discipline. ${ }^{18}$ Parmi les autres adeptes romands figure Alcide Jentzer (18491907), homme politique et professeur suppléant de gynécologie opératoire à la Faculté de médecine de Genève, qui inclut gymnastique et massages suédois dans sa pratique, d'après des méthodes inspirées par l'inventeur du massage gynécologique, le suédois Thure-Brandt. Jentzer et son assistant Maurice Bourcart (1864-1931), privat-docent à l'Université de Genève, fondent en 1893 l'Institut central de gymnastique pédagogique et médicale de Genève, où exercent également trois femmes diplômées de l'Institut royal de Stockholm, les sœurs Béronius et Mlle Ekelöf. Et tandis que Jentzer co-signe, avec les deux premières, un manuel de gymnastique pédagogique suédoise en 1894, Bourcart fonde en 1895 son propre cabinet de consultation et de clinique, où sont prodigués massages et gymnastique médicale suédoise; il supervise en outre au même endroit un institut de gymnastique suédoise pédagogique pour enfants et jeunes filles, dont les exercices sont donnés par Mlle Ekelöf, remplacée en 1903 par Mlle Bergman, également diplômée de Stockholm. Celle-ci traite notamment, sous la direction du médecin, les déviations de la taille, l'anémie, la constipation des enfants et des jeunes filles. ${ }^{19}$ Bourcart traduit aussi, en 1898, le traité de gymnastique médicale suédoise du professeur Anders Wide, lequel est directeur de l'Institut de gymnastique orthopédique de Stockholm, fondé par l'Etat suédois en 1827 à la suite de l'Institut central de gymnastique de Ling. Bourcart adjoint à la traduction du traité de Wide un chapitre sur ses propres recommandations en matière d'exercices de gymnastique suédoise dans les affections abdominales et gynécologiques. ${ }^{20}$

17 Ibid., 202-203.

18 Reymond 1893, 752. A noter que la Suisse alémanique est plus précoce, avec l'Institut de gymnastique médicale suédoise de Jakob J. Frey fondé en 1859, qui deviendra institut orthopédique puis sera repris par Lüning et Schulthess qui y pratiquent la mécanothérapie dès les années 1880: voir Rüttiman 1983.

19 Journal de Genève [encarts publicitaires], 4/10/1893, 24/08/1895, 12/10/1903, 18/01/1931.

20 Wide 1898. 
Cette corrélation entre gymnastiques suédoises pédagogique et médicale, centrées notamment sur les jeunes filles et, pour la gymnastique médicale, sur les affections féminines telles que la scoliose et les troubles gynécologiques, ajoutée au fait que quelques médecins s'adjoignent des professionnelles femmes, n'est pas le fruit du hasard. On se situe alors à une époque où l'exercice physique s'impose dans les conceptions hygiénistes pour l'ensemble de l'Europe. La gymnastique suédoise est alors tout particulièrement appréciée, car on cherche une gymnastique adaptée spécifiquement aux filles et aux femmes, pour lesquelles elle se présente comme la solution idéale. ${ }^{21}$ Une telle gymnastique, en effet, permet d'effectuer des mouvements de peu d'amplitude adaptés au corps féminin considéré comme fragile et sensible; une fonction que remplissent aussi les machines médico-mécaniques par des exercices ciblés selon les sexes: pour la gent féminine sont évités les levers de bras au-dessus des épaules, les écartements de jambes ou la position couchée, qui sont autorisés pour la gent masculine (fig. 2 et 3). Par ailleurs, Schwarzmann-Schafhauser a relevé l'incidence entre l'absence de la douleur dans la scoliose et le succès des instituts orthopédiques qui se centrent sur cette affection. ${ }^{22}$ Le corps des jeunes filles scoliotiques est donc particulièrement visé par la nouvelle thérapie orthopédique, et par les discours des spécialistes qui abondent sur le sujet dans le cadre de l'hygiène scolaire, autre champ médical en pleine essor du fait de la démocratisation de l'école dans le dernier tiers du XIX ${ }^{\mathrm{e}}$ siècle.

Ainsi, dans les débats portant sur l'étiologie de la scoliose, on ne s'étonne pas de retrouver l'orthopédiste Scholder, promoteur de la mécanothérapie, comme défenseur de la théorie - controversée - de la scoliose imputable à l'école, ou «scoliose scolaire». ${ }^{23}$ Il publie en effet en 1901 une étude novatrice sur Les déviations de la colonne vertébrale dans les écoles de Lausanne, qu'il co-signe avec le Dr Auguste Weith (1858-1947), remplaçant du premier médecin des écoles nommé pour la ville de Lausanne, lequel n'est autre que le troisième auteur de l'étude, Adolphe Combe (1859-1917), également professeur de clinique infantile à la Faculté de médecine de Lausanne. Réfutant l'argument médical bien établi d'une constitution féminine naturellement pathologique dans l'explication étiologique de la scoliose, les médecins lausannois mettent en avant des pratiques socioculturelles qui affectent tout particulièrement le développement des jeunes filles. D'une part, ils soutiennent que «c'est surtout chez les filles fortes et bien musclées, bien plus que chez

21 Je remercie V. Czáka pour ces informations, tirées de sa thèse de doctorat en cours: Czáka en cours.

22 Schwarzmann-Schafhauser 2004, 121.

23 Sur ce sujet et ce qui suit le concernant, voir aussi Kaba 2009. 
Fig. 2 et 3: Machines employées dans le système Zander: frottement des bras et suspension latérale (Scholder 1897, en annexe). Les brochures promouvant la mécanothérapie représentent des exercices différenciés selon les sexes: pour les patientes féminines, les mouvements sont de peu d'amplitude et effectués en position verticale; pour les hommes sont autorisés les mouvements amples des bras et des jambes, en position couchée.
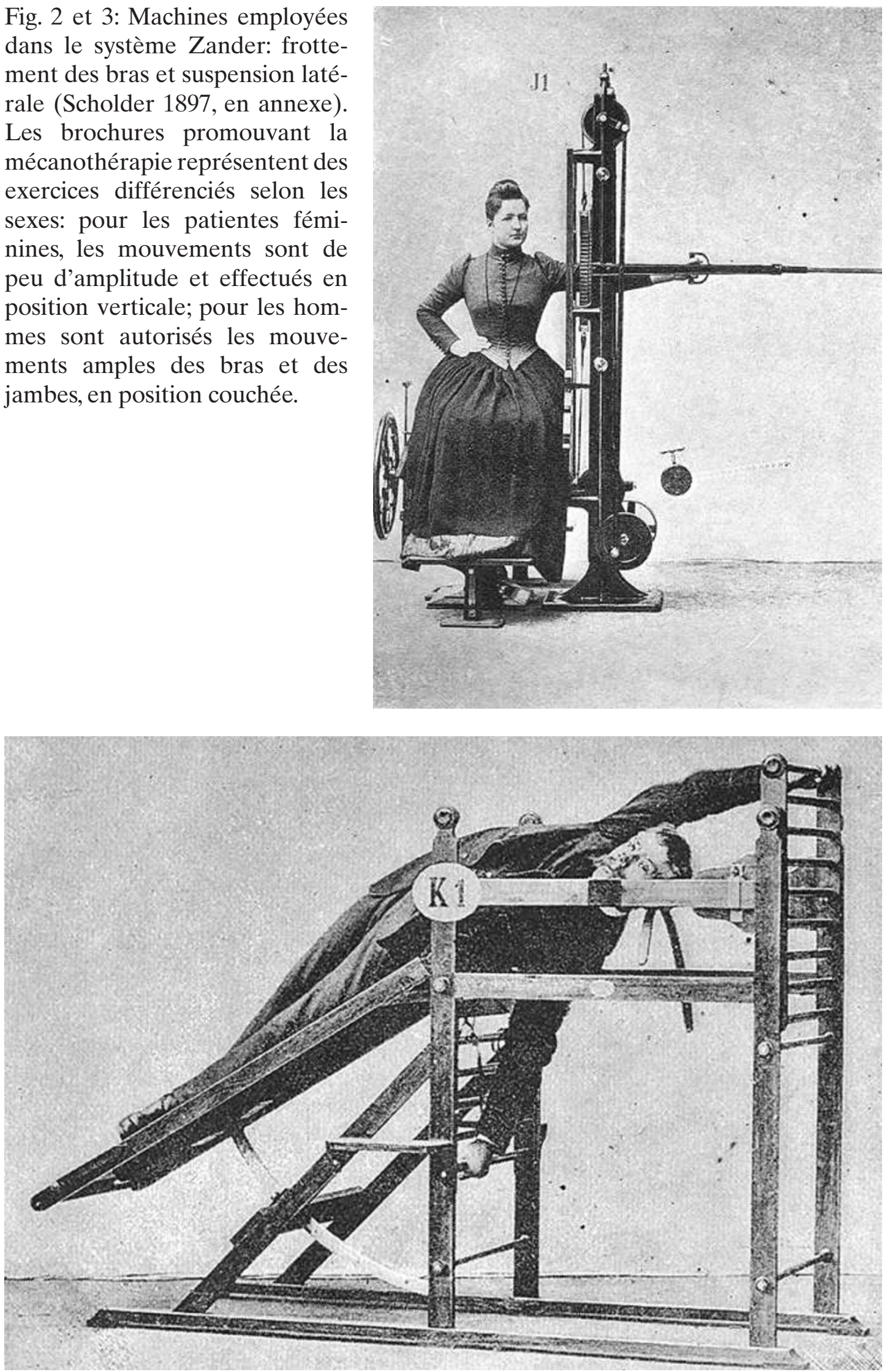
les garçons, que s'observe la scoliose. Or ce sont elles surtout qui portent à la maison leurs frères et sœurs et qui font les commissions». ${ }^{24} \mathrm{~A}$ cette charge asymétriquement répartie des divers fardeaux domestiques, qui concernent essentiellement les filles des couches populaires, s'ajoutent le port des livres d'école sur un même bras, ainsi que les jupons inégalement répartis des écolières qui s'installent au pupitre en entrant d'un côté du banc. D'autre part, les médecins relèvent dans leur étude une surreprésentation des scolioses chez les filles des écoles supérieures, du fait des cours qui leur sont spécifiquement dispensés tels que piano, broderie, peinture, qui viennent s'ajouter aux fatigues de l'école; c'est pourquoi, estiment-ils, chez celles-ci, «le nombre des scolioses augmente avec celui des années». ${ }^{25}$ Ici, les médecins lausannois condamnent donc le cumul d'activités présentées, du point de vue social, comme le parangon de la civilisation moderne occidentale - les jeunes filles de bonne famille sont instruites afin de devenir des épouses et mères dignes de la société Belle Epoque -, mais considérées, du point de vue médical, comme nuisibles pour leur santé. Les activités domestiques et scolaires des filles des classes populaires, qui n'accèdent pas à l'école supérieure, étant autrement néfastes comme on l'a vu.

Car, outre l'aspect esthétique disgracieux de la scoliose pour la taille des jeunes filles, on estime alors que l'affection comporte des risques biologiques inhérents à la gent féminine, le lien entre corps dévié et procréation étant notamment formulé par les médecins lausannois: «Si l'affection n'est pas traitée, le rétrécissement du bassin peut avoir des conséquences désastreuses pour les femmes scoliotiques qui ne craignent pas le mariage.» ${ }^{26}$ (fig. 4) Il s'agit là d'un argument récurrent du discours hygiéniste, révélateur du rôle sexué attribué au corps féminin centré sur sa fonction biologique de réceptacle des futures générations, que la scoliose mettrait en péril. C'est pourquoi la consultation d'un spécialiste sera plus systématique pour les filles, ce que confirme un tableau statistique des scolioses dénombrées chez une dizaine d'orthopédistes européens - au nombre desquels figure Scholder -, qui donne une moyenne de $85,8 \%$ de filles scoliotiques ayant consulté pour $14,2 \%$ de garçons. ${ }^{27}$ On a aussi relevé plus haut la faible proportion de garçons scoliotiques traités à l'Institut médico-mécanique de Reymond (8 garçons sur 66 patient·e.s). La question est alors de savoir si les jeunes filles des classes populaires et des classes aisées ont également accès au traitement de la mécanothérapie proposé par certains orthopédistes.

24 Scholder/Weith/Combe 1901, 49.

25 Ibid., 35.

26 Ibid., 23.

27 Ibid., 34. 
Fig. 4: Jeune fille scoliotique des écoles de Lausanne (Scholder/Weith/Combe 1901, 24). La scoliose est particulièrement contrôlée chez les filles pour des raisons d'ordre esthétique ainsi que médical: dans les cas les plus graves, on estime que le rétrécissement du bassin peut porter préjudice à une future grossesse.

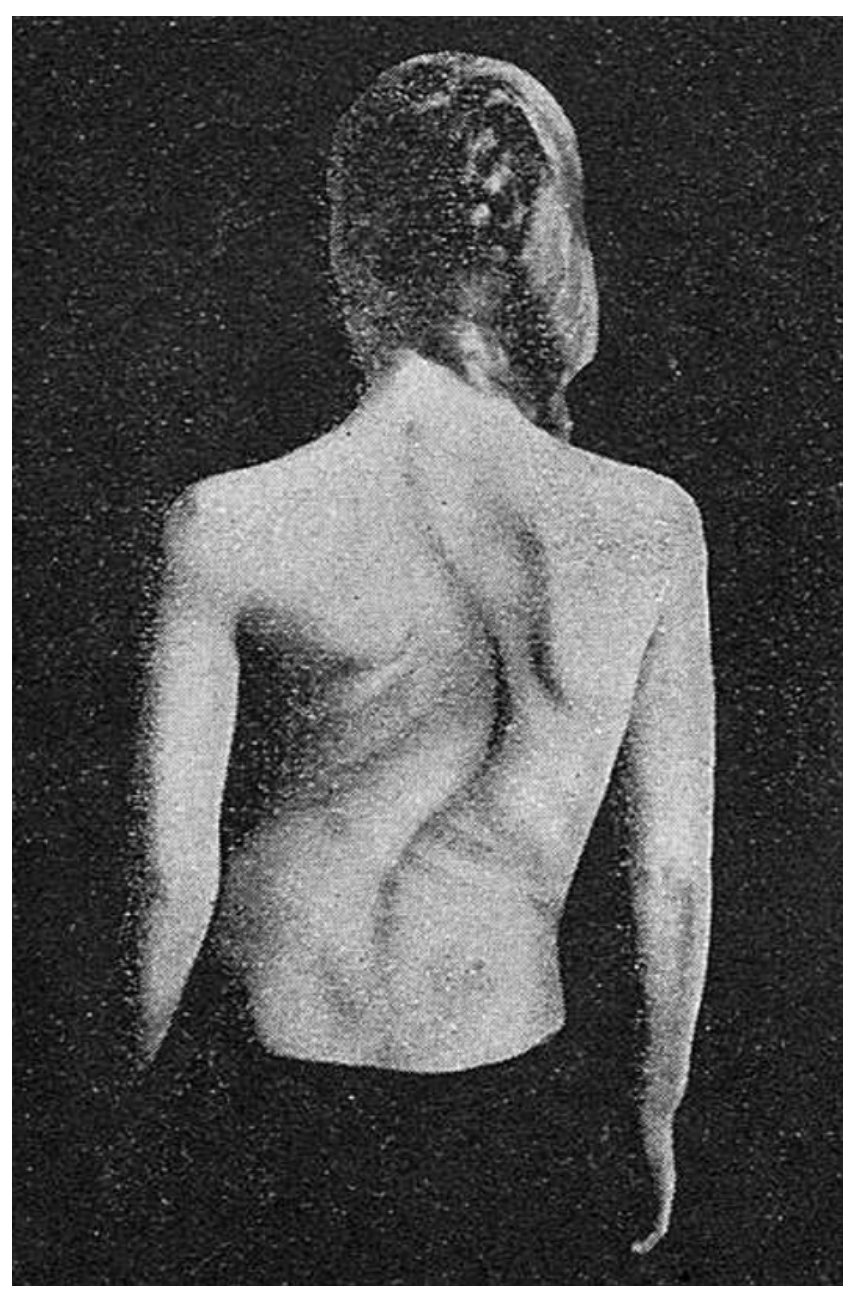

\section{La clientèle de la mécanothérapie: des jeunes filles scoliotiques de bonne famille avant tout?}

La mécanothérapie, qui se développe surtout dans les villes privilégiées par le tourisme et l'académisme médical, est-elle un traitement accessible à toutes et tous ou une thérapie de luxe? Les sources restent muettes sur le sujet du niveau socioprofessionnel des patient.e-s fréquentant les instituts médico-mécaniques. Toutefois, les tarifs du traitement indiqués par Reymond en 1898 permettent une comparaison avec les salaires de l'époque. Ainsi, la séance de mécanothérapie - qui consiste dans l'emploi de dix à douze machines - revient à 5 francs suisses (ci-après frs), avec des réductions en cas de séances répétées: par exemple, 20 et 30 frs pour respectivement cinq et dix séances par semaine pendant un mois. ${ }^{28}$ Or, les employé·e·s du secteur

28 Reymond 1898, 4 page du prospectus inséré. 
secondaire (industries textile et chimique, transports, construction, alimentation) du plateau suisse alémanique ont un salaire moyen hebdomadaire de 24 frs en 1898, assez semblable aux 80 à 120 frs mensuels que gagnent une téléphoniste de la poste ou une vendeuse suisse vers $1900 .{ }^{29}$ Autant dire que l'accès à l'institut reste difficile pour ces travailleuses et travailleurs peu fortunés, pour lesquels une seule séance reviendrait à soustraire environ un cinquième du salaire hebdomadaire. De plus, une remarque des chirurgiens français Paul Berger et S. Banzet, auteurs d'un traité de Chirurgie orthopédique paru en 1904, vient confirmer l'hypothèse d'une thérapie peu accessible:

On trouvera plus loin, spécialement à l'occasion du traitement des déviations vertébrales, la description de types variés de ces machines [médico-mécaniques] et de leur mode d'emploi. Leur nombre s'est beaucoup accru et leur construction s'est notablement perfectionnée, mais leur principal inconvénient est de ne pouvoir être utilisés que dans des installations spéciales qui ne sont pas à la portée de tous les malades. ${ }^{30}$

On ignore si le manque d'accessibilité se situe au niveau financier ou géographique, mais on peut supposer que les deux hypothèses sont vraies. On notera par ailleurs, chez ces auteurs également, l'indication de la grande proportion de machines centrées surtout sur les déviations de la colonne vertébrale. Ce dernier point nous ramène à la question de la clientèle qui consulte surtout pour les cas de scoliose.

Davantage qu'un phénomène médical, il faut comprendre cette focalisation sur la scoliose comme un fait social et culturel. Etudiant l'essor de l'orthopédie en Allemagne, Schwarzmann-Schafhauser s'est interrogée sur les raisons pour lesquelles le pied bot, qui vaut comme prototype du mal orthopédique au début du XIX ${ }^{\mathrm{e}}$ siècle et pour lequel le traitement obtient de meilleures chances de succès, ne s'est pas imposé comme la scoliose qui, bien que se laissant difficilement traiter, est la plus représentative chez les patient-e.s fréquentant les institutions orthopédiques. Selon elle, il faut expliquer la nette hausse des demandes de la population pour le traitement de la scoliose par le fait que celui-ci concerne surtout les classes fortunées et non les classes populaires, pour lesquelles les soins relèvent d'une offre émanant d'initiatives privées (organisations sociales, religieuses) et non d'une demande spécifique de la clientèle. ${ }^{31}$ C'est aussi pourquoi les institutions de la seconde moitié du siècle destinées aux enfants des classes indigentes atteints d'affections orthopédiques concernent une multitude d'autres maladies que la simple scoliose idiopathique, les philanthropes investissant

29 Siegenthaler/Ritzmann-Blickenstorfer 1996, 448; Bühlmann/Zatti 1992, 248.

30 Berger/Banzet 1904, 10.

31 Schwarzmann-Schafhauser 2004, 116-117. 
dans les maladies particulièrement graves et invalidantes pour les futures forces économiques du pays. Ce phénomène est confirmé par l'étude allemande de Thomann, qui s'interrogeait sur l'existence d'une orthopédie au $\mathrm{XIX}^{\mathrm{e}}$ siècle en tant que spécialité médicale réservée aux personnes aisées: son analyse de l'Institut orthopédique de Würzburg de la famille Heine, père et fils, fondé en 1816 et maintenu tout au long du XIX ${ }^{\mathrm{e}}$ siècle, montre que l'établissement traitait à ses débuts une majorité de scolioses chez une clientèle plutôt aisée, puis s'est destiné peu à peu à une clientèle indigente affectée de troubles orthopédiques sévères. ${ }^{32}$

Ainsi, la scoliose relèverait de l'exagération d'un souci des parents de l'élite sociale pour cette affection chez les jeunes filles. Comme le souligne Schwarzmann-Schafhauser, il y aurait un impératif moral à consulter, une culpabilisation de la part de certains médecins, tandis que d'autres constatent que les consultations sont souvent non justifiées ou prophylactiques, parfois aussi voulues par les jeunes filles, qui simuleraient la scoliose. Mais, si quelques rares médecins avancent l'argument d'une maladie psychique, dans le sens d'une hystérie des jeunes filles ou d'une angoisse exagérée des parents - sorte de phénomène de mode féminin également décrit pour la chlorose,$-{ }^{33}$ beaucoup sont convaincus de l'aspect organique de la scoliose. Jusqu'au tournant du siècle, on ne distingue pas entre vraie scoliose structurelle et position incorrecte scoliotique, laquelle a valeur de maladie. C'est seulement dans les années 1910 que germera la prise de conscience que la scoliose est une variété inoffensive dans le spectre du normal ou de la posture déviée, sans anomalie du squelette. ${ }^{34}$

\section{Essor et déclin d'une thérapie technique féminine et élitiste}

L'inquiétude «élitiste» pour la scoliose est donc une caractéristique de cette affection, comme le montre son incidence sur l'essor de la mécanothérapie, produit des avancées techniques qui culminent à la Belle Epoque. L'ensemble des prescriptions de cette thérapie technique va en outre dans le sens d'une clientèle surtout féminine, en continuité logique de ce qui a été conçu par les partisans de la gymnastique suédoise. On en rappellera ici les principales raisons. Premièrement, la mécanothérapie est centrée majoritairement sur

32 Thomann 1989.

33 Voir par exemple l'étude sur la chlorose chez les adolescentes démontrant les interactions complexes entre la physiologie des jeunes filles, la médecine et la vie familiale et sociale, dans Brumberg 1984.

34 Schwarzmann-Schafhauser 2004, 118-121. 
la scoliose, laquelle implique des facteurs biologiques et socioculturels inhérents au genre féminin. Deuxièmement, la nouvelle thérapie répond aux impératifs d'une gymnastique douce, adaptée au corps féminin alors perçu comme fragile et sensible. Troisièmement, il semblerait que, à une époque où la médecine post-pasteurienne et «scientifique» connaît un essor indéniable, de nombreux patient.e.s se détournent de cette voie pour expérimenter une médecine clairement alternative. Le tournant du siècle voit un fort engouement pour les thérapies physiques, développées en cliniques privées et en sanatoriums, qui sollicitent les éléments naturels (eau, air, soleil) et allient souvent diététique et psychothérapie; des thérapies auxquelles on peut ajouter la gymnastique suédoise et la mécanothérapie, qui attirent alors majoritairement des femmes de milieux aisés. ${ }^{35}$

Or, malgré l'apparent engouement pour la mécanothérapie, les instituts spécifiques à cette pratique ne connaissent pas d'essor notable après la Belle Epoque. Du temps même des principaux promoteurs de la mécanothérapie, un recul d'attractivité est déjà signalé, découlant, d'une part, du manque de soutien académique pour ce que Reymond désigne alors sous le terme de «physico-thérapie» - qui deviendra «physiothérapie»-, incluant la gymnastique médicale. ${ }^{36}$ D'autre part, le médecin observe un certain dédain de la clientèle régulière, qu'on peut attribuer à un système probablement victime de sa nouveauté et de sa modernité:la haute technicité mécanothérapique a pu faire croire à un traitement accéléré, le machinisme industriel encourageant cette conception du toujours mieux et toujours plus rapide; or, le corps reste tributaire de sa matérialité, dont la «réparation» accélérée ne peut être assurée par aucune machine, aussi sophistiquée soitelle, la répétition régulière des exercices sur la durée restant le plus souvent la meilleure alliée de la guérison. Et alors qu'aucun chiffre n'est donné quant aux résultats effectifs obtenus par le traitement mécanothérapique, Reymond impute les échecs de celui-ci à son abandon précoce par les patient·e.s ... impatient·e.s. Dès lors, l'institut médico-mécanique de l'orthopédiste genevois, quitté par celui-ci en 1902 pour cause de maladie, sera repris successivement par deux médecins pour fermer ses portes en 1916. Tandis que le décès de Scholder, survenu en 1918, mettra aussi un terme à l'institut lausannois; à la même adresse demeurera toutefois la veuve de l'orthopédiste et leur fils, Jean-Charles Scholder (1891-1958), qui y recevra sa clientèle en tant que chirurgien orthopédiste dès 1939. ${ }^{37}$

35 Je l'ai aussi montré pour le sanatorium du Dr Bircher-Benner, inventeur du Bircher müesli, qui allie diététique, gymnastique, hydrothérapie, etc.: Kaba 2010.

36 Reymond 1901,192.

37 Indicateurs vaudois et genevois, bottins, annuaires commerciaux 1897 et ss. 
Zichner, Rauschmann et Thomann ont expliqué le déclin de la thérapie de Zander vers la période du premier conflit mondial par le coût élevé et le manque de mobilité des appareils. Il semblerait qu'il ait fallu attendre les conséquences de la guerre pour constater un regain d'intérêt pour la gymnastique médicale, dans ce qui devient les thérapies de la réhabilitation: le nombre important de blessés pose de nouveaux défis à la chirurgie orthopédique, tandis que la gymnastique médicale gagne en signification. Le manque de thérapeutes qualifiés aurait alors favorisé l'ouverture, au cours $\mathrm{du} \mathrm{XX}^{\mathrm{e}}$ siècle, de nouvelles écoles de formation à la gymnastique médicale, pratiquée surtout dans le cadre de la physiothérapie. ${ }^{38}$ Welti a quant à elle relevé, pour la Suisse alémanique, l'existence de deux mouvances dans la pratique du massage et de la gymnastique médicale, selon qu'on se forme en Suède ou en Allemagne; tandis qu'en Allemagne, la concurrence entre les gymnastiques suédoise et allemande se solde par la victoire de la seconde, plus populaire. ${ }^{39}$ La situation semble quelque peu différente en Suisse romande, puisque la gymnastique suédoise se maintient sous sa forme non mécanisée au sein de la gymnastique pédagogique des milieux scolaires, les principaux initiateurs romands, on l'a vu, s'étant formés surtout en Suède. Mais sur le marché médical saturé, où les médecines naturelles parallèles connaissent un regain d'intérêt, les thérapies physiques, telles que la mécanothérapie, se maintiennent dans des niches plus discrètes qu'autrefois, occupant une place parmi d'autres thérapies dans des institutions nouvellement créées destinées à la prise en charge médicale du corps infirme. Une filiation s'établira également entre les machines médico-mécaniques et celles qui perceront plus tard dans le $\mathrm{XX}^{\mathrm{e}}$ siècle, sous une forme adaptée, dans les salles de musculation ou de remise en forme.

Il faut noter enfin que les débats médicaux sur la scoliose perdent en intensité une fois passé le seuil du XX $\mathrm{X}^{\mathrm{e}}$ siècle. Guillaume l'explique par le fait que les priorités hygiénistes ont changé: c'est le temps de la grande hantise antituberculeuse et les recherches bactériologiques apportent de nouvelles connaissances sur le processus de diffusion des maladies infectieuses. ${ }^{40} \mathrm{La}$ lutte et surtout la prévention des épidémies deviennent la dimension majeure de l'hygiène médicale; le spectre d'autres pathologies plane dès lors sur le corps des jeunes générations.

38 Zichner/Rauschmann/Thomann 1999, 76.

39 Welti 1997; Schwarzmann-Schafhauser 2004, 127.

40 Guillaume 2005, 225. 


\section{Bibliographie}

Berger, Paul/S. Banzet, Chirurgie orthopédique (Paris 1904)

Bonna, Auguste-E., «Institut médico-mécanique du Dr C. Reymond, à Genève», La Patrie Suisse 141 (1899) 45-46

Brumberg, Joan Jacobs, «Chlorotic girls, 1870-1920: A historical perspective on female adolescence», in: Judith Walzer Leavitt (éd.), Women and Health in America (Madison 1984) 186-195

Bühlmann, Yvonne/Kathrin Zatti, Frauen im schweizerischen Telegrafen- und Telefonwesen 1870-1914 (Zürich 1992)

Campagne, Florence, «Dossiers santé: Le mal de dos ( $2^{\mathrm{e}}$ partie): La scoliose», www.caducee.net/ DossiersSpecialises/rhumatologie/mal-de-dos2.asp (juillet 2000)

Czáka, Véronique, La gymnastique scolaire comme lieu de construction du genre: l'exemple de la Suisse romande (1860-1920), thèse de doctorat en histoire inscrite à l'Université de Lausanne (en cours)

Donzé, Pierre-Yves, L'ombre de César. Les chirurgiens et la construction du système hospitalier vaudois (1840-1960) (Lausanne 2007)

Guillaume, Pierre, «L'hygiène à l'école et par l'école», in: Patrice Bourdelais/Olivier Faure (éds), Les nouvelles pratiques de santé. XVIII $-X X^{e}$ siècles (Paris 2005) 213-226

Hefti, Fritz et al., Kinderorthopädie in der Praxis (Berlin 1998)

Indicateurs vaudois et genevois, bottins, annuaires commerciaux (1897 et ss)

Journal de Genève [encarts publicitaires], 4/10/1893, 24/8/1895, 12/10/1903, 18/1/1931

Kaba, Mariama, «Exigences du corps et déficiences physiques chez les filles et les garçons. Contribution aux gender \& disability studies», in: Anne Dafflon Novelle (éd.), Filles - garçons. Socialisation différenciée? (Grenoble 2006a) 203-222

Kaba, Mariama, «Quelle place pour une perspective genre dans la disability history? Histoire du corps des femmes et des hommes à travers le handicap», Traverse. Revue d'histoire 3 (2006b) 47-60

Kaba, Mariama, «Quand éducation rime avec déviation. La scoliose chez les filles et les garçons comme enjeu de la médecine scolaire à Lausanne (fin $\mathrm{XIX}^{\mathrm{e}}$-début $\mathrm{XX}^{\mathrm{e}}$ siècle)», Revue historique vaudoise 117 (2009) 89-101

Kaba, Mariama, «Les premières décennies du sanatorium Bircher de Zurich à la lumière des dossiers de patients», dans: Eberhard Wolff (Hrsg.), Lebendige Kraft. Max Bircher-Benner und sein Sanatorium im historischen Kontext (Baden 2010) 78-95

Kaba, Mariama, Malades incurables, vieillards infirmes et enfants difformes. Histoire sociale et médicale du corps handicapé en Suisse romande (XIX $X^{e}$-début XX $X^{e}$ siècle), thèse de doctorat en histoire de l'Université de Lausanne (Lausanne 2011)

Monet, Jacques, La naissance de la kinésithérapie (1847-1914) (Paris 2009)

Rang, Mercer, The Story of Orthopaedics (Philadelphia 2000)

Reymond, Camille, «Gymnastique suédoise», Revue médicale de la Suisse romande (1893) $685-695$ et $737-753$

- Institut médico-mécanique et orthopédique de Genève et la mécanothérapie de Zander. Définition - Indications (Genève 1898)

- «Quelques résultats de la thérapeutique par les machines de Zander, à l'Institut médicomécanique de Genève. Communication faite à la Société médicale de Genève le 6 février 1901», Revue médicale de la Suisse romande (1901) 191-204

Rieder, Philip, Anatomie d'une institution médicale. La Faculté de médecine de Genève (18761920) (Lausanne 2009)

Rüttimann, Beat, Wilhelm Schulthess (1855-1917) und die Schweizer Orthopädie seiner Zeit (Zürich 1983)

Scholder, Charles, La mécanothérapie: sa définition et ses indications d'après le système Zander (Lausanne 1897)

Scholder, Charles, /Auguste Weith/Adolphe Combe, Les déviations de la colonne vertébrale dans les écoles de Lausanne (Zurich 1901)

Schwarzmann-Schafhauser, Doris, Orthopädie im Wandel. Die Herausbildung von Disziplin und Berufsstand in Bund und Kaiserreich (1815-1914) (Stuttgart 2004) 
Siegenthaler, Hansjörg/Heiner Ritzmann-Blickenstorfer (éd.), Statistique historique de la Suisse (Zurich 1996)

Thomann, Klaus-Dieter, «Orthopädie im 19. Jahrhundert: Eine medizinische Spezialdisziplin für die Wohlhabenden?», Medizin, Gesellschaft und Geschichte 8 (1989) 27-62

Valentin, Bruno, Geschichte der Orthopädie (Stuttgart 1961)

Vigarello, Georges, Le corps redressé. Histoire d'un pouvoir pédagogique (Paris 2001)

Welti, Sabine, Massage und Heilgymnastik in der ersten Hälfte des 20. Jahrhunderts: zur Professionalisierung der Physiotherapie (Wabern 1997)

Wide, Anders, Traité de gymnastique médicale suédoise. Traduit, annoté et augmenté de plusieurs chapitres sur les affections abdominales et gynécologiques par le $\mathrm{Dr}$ M. Bourcart (Bâle/ Genève 1898)

Zichner, Ludwig/Michael A. Rauschmann/Klaus-Dieter Thomann (Hrsg.), Orthopädie. Geschichte und Zukunft (Darmstadt 1999) 